\title{
APPLICATION OF AN EULER SOLVER TO SELECTED PROBLEMS IN FLIGHT DYNAMICS
}

\author{
J. Sznajder, J. Żółtak \\ Institute of Aviation, Warsaw. 110-114 Al. Krakowska PL-02-256 Warsaw. Poland \\ E-mail: jsznaj@ilot.edu.pl \\ Received 0611 2006, accepted 11062007
}

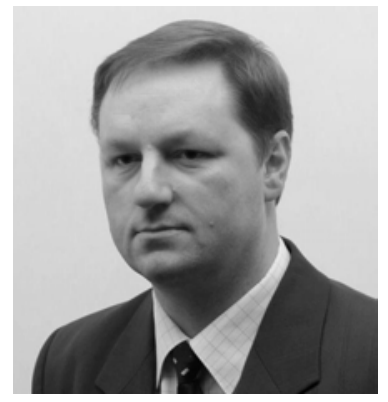

Janusz SZNAJDER graduated in 1993 from Warsaw University of Technology and is currently employed at the Institute of Aviation in Warsaw. He obtained a doctoral degree from the Warsaw University of Technology in 1999. He participated in several industrial and research projects conducted at the Institute of Aviation, including European projects from the 5th and 6th Framework Programme. He also coordinated two research grants of the Ministry of Education and Science. His publications concentrate on aircraft dynamics, stability, and aerodynamic loads.

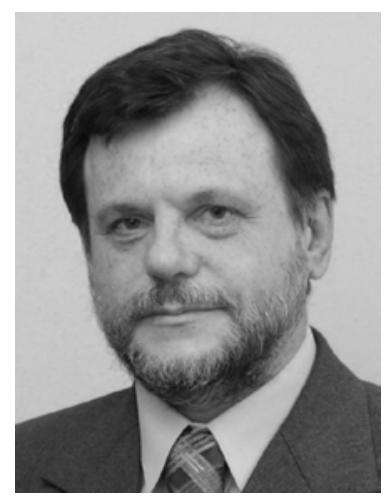

Dr Jerzy ŻółTAK has led the Numerical Aerodynamic and Flight Dynamics Group at the Aviation Institute in Warsaw, Poland since 1992. Earlier he was the senior researcher at the High Speed Wind Tunnel Laboratory (1980-1992) and an associate researcher at Warsaw University of Technology (1995-1997) He was also a visiting researcher at the Technical Engineering Department of the University of Manchester Institute of Science and Technology (for nine months in 1996).

His interests include simulation of transonic flows, steady and unsteady aerodynamics, fluid structure interaction, and parallel computing.

Abstract. Several applications of a Euler solver with the formulation of the flow equations in the noninertial reference system with steady and unsteady flow analysis are presented. The steady-flow applications include determination of aerodynamic derivatives with respect to angular velocity and analysis of vortical flow over a delta wing at high angles of attack with the determination of aerodynamic coefficients and analysis of vortex breakdown. The unsteady flow analysis is applied in the simulation of a rapid manoeuvre for the determination of unsteady forces. The results of this simulation are compared with results of simulations using steady-flow approximation in order to assess the advantages of unsteady flow analysis in the simulation of aircraft manoeuvres.

Keywords: Euler solver, steady and unsteady flow, flight dynamics, simulation.

\section{Introduction}

The development of highly manoeuvrable aircraft, operating at a wide range of angles of attack, including angles of attack beyond the stall limit, is a reason to search for new methods to analyse aircraft dynamics and stability. The phenomena challenging the classical method of analysing of aircraft dynamics, based on aerodynamic derivatives, include:

- $\quad$ vortex flow at high angles of attack and instability of the vortex system, leading to sudden changes in the longitudinal and lateral aerodynamic derivatives,
- $\quad$ sudden manoeuvres of aircraft with small positive, or negative stability margins, leading to the rise of high unsteady forces,

- strong aerodynamic interference during store separation.

Significant improvement in the analysis of aircraft dynamics can be obtained through the wider application of CFD codes in flight dynamics. Considering the computational capabilities of the computers most widely available for engineering applications at the present time (PC platform), the most effective CFD tools for 
application in flight dynamics are Euler solvers. The numerical solutions of Euler equations predict many flow phenomena important for today's highly manoeuvrable aircraft like shock waves or vortex lift and can be used for the analysis of steady and unsteady flow. The steady flow applications of Euler solvers include the determination of aerodynamic characteristics and aerodynamic derivatives necessary for the analysis of aircraft stability, and the unsteady flow analysis can be used for the concurrent solution of the aircraft equations of motion and flow equations in cases with high unsteady aerodynamic loads. An example of such an analysis was presented by S.M. Hitzel, E. van der Weide, U. Tremel, H. Rieger in which the steep descent of a delta-winged aircraft at high angles of attack was analysed [4]. Due to the complexity of the problem, resulting from the determination of the flow around a three-dimensional, real aircraft, the solution was highly parallelized, with the application of up to 256 processors.

In this paper, examples of steady and unsteady-flow applications of an Euler solver in flight dynamics are presented. The steady-flow cases include computation of aerodynamic derivatives with respect to angular derivatives in two-dimensional flow and analysis of vortex lift of a low-aspect ratio delta-wing. The analysis, including the determination of the position of vortex breakdown, was conducted using the presented, implicit Euler solver with upwind discretisation of flux vectors. The results were compared to the results of an earlier work with a centred scheme and additional dissipative terms stabilizing the solution.

An application of the analysis of unsteady flow for the concurrent determination of the aerodynamic loads and the simulation of aircraft manoeuvre is presented for a simplified aircraft configuration. It was shown that concurrent solution of the aircraft equations of motion and flow equations may supplement the classic model of aircraft dynamics, in which the effects of flow unsteadiness are approximated using the force and moment derivatives with respect to aircraft acceleration. The advantage of the approach presented is the possibility of obtaining time-accurate solution of unsteady flow. The most significant differences between the results of the classic model of aircraft dynamics and the presented model are in the values of peak loads, especially for low values of stability margin, and they can be observed even for simple, traditional aircraft configurations.

\section{Description of the solver}

For the application of Euler flow equations in flight dynamics, the most convenient is the formulation of equations in a non-inertial reference system. The main reason for using such a formulation is that in a noninertial, coordinate system attached to the aircraft it is possible to use a standard, zero-normal velocity boundary condition on the aircraft surface, while the use of an inertial reference system would require the implementation of moving-wall boundary conditions. In an inertial reference system also additional computational load is needed in each time step for updating the position and orientation of the aircraft. For the solution of the flow equations, the finite volume method was chosen, and the equations of balance for a control volume have the form [7]:

$$
\frac{d}{d t} \int_{V(t)} U d V+\int_{S(t)} \vec{n} \cdot \mathrm{F} d S d t-\int_{V(t)} \mathrm{G} d V=0 .
$$

where:

$\mathrm{V}(\mathrm{t})$ - the control volume,

$\mathrm{S}(\mathrm{t})$ - the boundary surface of the control volume,

$\mathrm{U}$ - vector of conservative variables,

$\vec{n}$ - vector normal to $\mathrm{S}(\mathrm{t})$, oriented outwards,

$\mathrm{F}$ - tensor of flux through the boundary $\mathrm{S}(\mathrm{t})$,

$\mathrm{G}$ - source vector defined by the equation (4)

The state of the flow is determined by the vector of conservative variables:

$$
U=\left[\rho, \rho u_{x}, \rho u y, \rho u_{z}, e\right]^{\mathrm{T}}
$$

The flux tensor is described by the following formula:

$$
F=\left[\left[\begin{array}{c}
\rho u_{x} \\
\rho u_{x}^{2}+p \\
\rho u_{x} u_{y} \\
\rho u_{x} u_{z} \\
(e+p) u_{x}
\end{array}\right],\left[\begin{array}{c}
\rho u_{y} \\
\rho u_{y} u_{x} \\
\rho u_{y}^{2}+p \\
\rho u_{y} u_{z} \\
(e+p) u_{y}
\end{array}\right],\left[\begin{array}{c}
\rho u_{z} \\
\rho u_{z} u_{x} \\
\rho u_{z} u_{x} \\
\rho u_{z}^{2}+p \\
(e+p) u_{z}
\end{array}\right]\right] .
$$

where $u_{x}, u_{y}, u_{z}$ are Cartesian components of velocity vector in no inertial frame, $\rho$ is density, $e$ is total energy per unit volume, and $p$ is pressure.

The vector $\mathrm{G}$ is defined as $[5,6]$ :

$$
\vec{G}=\left[\begin{array}{c}
0 \\
\rho \vec{\Omega}-2 \rho(\vec{\omega} \times \vec{W}) \\
\rho \vec{\Omega} \cdot \vec{u}
\end{array}\right] .
$$

where $\vec{\Omega}$ is a vector of "pseudo-forces" defined by:

$$
\vec{\Omega}=-\vec{\omega} \times(\vec{\omega} \times \vec{r})-\frac{d \vec{\omega}}{d t} \times \vec{r}-\frac{d \vec{W}}{d t} .
$$

where $\vec{\omega}$ is angular velocity vector, $\vec{r}$ is vector position, and $\vec{W}$ is the velocity of the non inertial frame in the inertial frame. Vector $\vec{\Omega}$ contains all effects of non-inertial motion, except for the Coriolis term, $-2 \omega \times \vec{W}$. The system of the flow governing equation is completed by the equation of the state of perfect gas. The simple implicit discretisation of equation (1) can be written as:

$$
\begin{aligned}
& \delta_{t}^{-}\left(V U_{l}\right)^{n+1}+\ldots \\
& \ldots+\sum_{i=1}^{N S}\left[\vec{n}_{i} \cdot F\left(U_{i}^{n+1}\right)\right] S_{i}-V G\left(U_{i}^{n+1}\right)=0 .
\end{aligned}
$$


where $\delta_{t}^{-}$denotes backward second order time approximation [7, 8].The MUSCL extrapolation scheme is used for the calculation of values of flow wariables on the cell walls.

The fluxes are calculated using the flux vector splitting in the form presented in [2] source.

The system of nonlinear algebraic equations (6) is solved by a Newton-type method [7, 8].

\section{Parallelization}

The Euler solver presented was implemented under a Linux operating system on a PC platform. Due to the limited computational capabilities of a single PC computer, the solution process had to be parallelized. The parallelization starts from the division of the grid into files containing data of single blocks of grid. This solution allows the grid blocks to be read by different processes depending on the number of processors available. A single processor manages each computational process. Each grid file contains a block interface section, which is composed of the data of cells located on block walls and links to cells adjacent to them, but belonging to the neighbouring blocks. After reading grid files, each process creates its own interface table, which allows the process to group the links into two categories: those belonging to cells within blocks read by the process and to cells read by other processes. The exchange of information between cells belonging to the same process is managed within the computer's operational memory, and the exchange of information between cells belonging to different processes is conducted using functions from Message Passing Library implemented in the Fortran programming language.

The solution of equation (6) is divided between each computational process so that each process solves the equation on the portion of the grid it has read into the operating memory. The exchange of information on the boundaries of grids belonging to different processes is conducted in each step of the Newton iterations, ensuring the coupling of all the processes.

\section{Steady flow applications-determination of stability derivatives and flow at high angles of attack}

The most obvious applications of the CFD solver in steady-flow analysis is the determination of aircraft aerodynamic characteristics and stability derivatives. The formulation of the flow equations in a non-inertial reference system enables efficient determination of the stability derivatives with respect to angular velocities, which may be used as a test of the method. An example of such an analysis is presented in figures 1 and 2, which show the values of the $c_{L}$ and $c_{M}$ coefficients of a NACA 0012 airfoil in rotating flight against different values of non-dimensional pitch rate. The results were compared with the results of viscous code, based on N-S equations also formulated in non-inertial reference frame
[5]. A close agreement between the results compared was obtained.

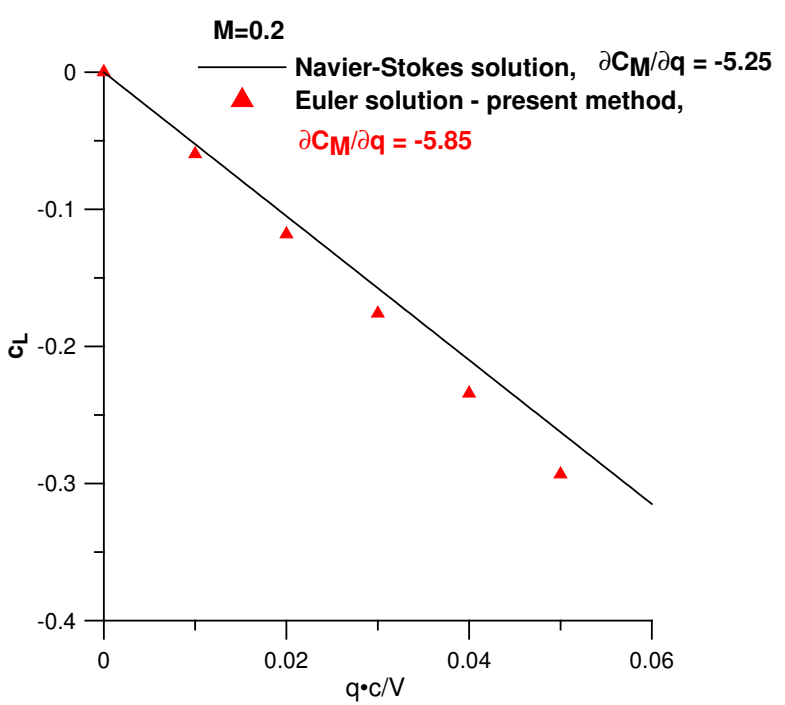

Fig 1. Lift coefficient of NACA 0012 airfoil vs. non-dimensional pitch rate

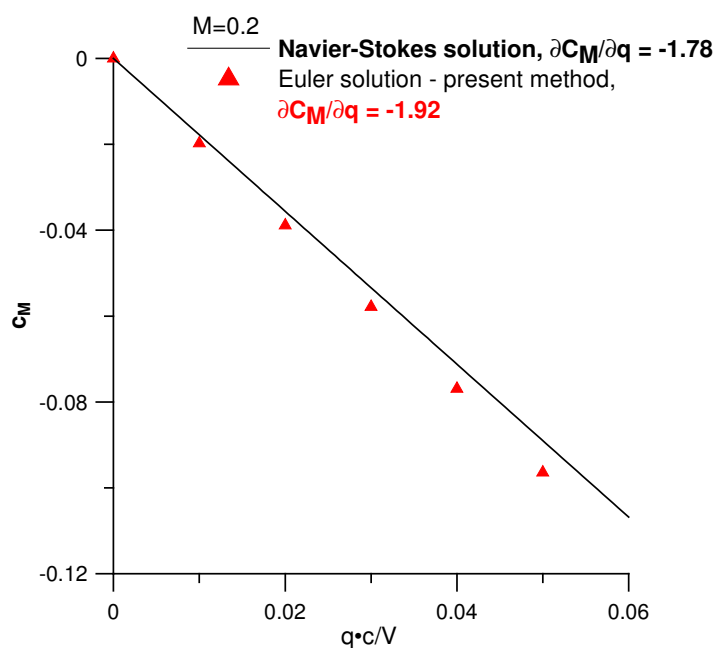

Fig 2. Moment coefficient of NACA0012 airfoil vs. nondimensional pitch rate

\section{High angle of attack aerodynamics}

In the aerodynamics of highly manoeuvrable aircraft, vortex lift occurring at high angles of attack is a very important phenomenon, enabling rapid turns and improving performance in other fields, such as fast takeoff or stabilization of flow on the tail surface through strake-type vortex generators. The vortex flow responsible for vortex lift at high angles of attack is the subject of intensive numerical and experimental investigations concerning the stability of the vortex system and its influence on the flow on lifting surfaces.

Currently, most of the CFD analysis of the flow at high angles of attack is conducted using the viscous flow model with turbulence modelling, which provides a very realistic flow solution, with all details of the complicated flow pattern such as the separation and the creation of the main vortex on the swept leading edge, secondary and tertiary separations and vortices on wing segments, and 
vortex breakdown. However, the main features of the vortex flow on highly swept wing segments at high angle of attacks, such as the creation and strength of the main vortex and the location of vortex breakdown can be analyzed through the numerical solutions of Euler equations. It is possible because the numerical dissipation, which is inherently present in the schemes of spatial discretisation, emulates to some degree the viscosity in a real flow.

The comparisons of Euler solutions of flow on lowaspect ratio delta wings with the solutions of Reynoldsaveraged Navier-Stokes equations have shown that the value of vortex lift in Euler solutions is very close to the results for viscous flow. The position of vortex breakdown and changes due to changes in the angle of attack also correlates well with the results obtained for viscous flow and with results of experimental investigations. The most important difference between the solutions of Euler and Navier-Stokes equations for low aspect-ratio wings and high angles of attack is lack of secondary art tertiary vortices in the vicinity of the main vortex, which appear as results of interactions between the main vortex and the boundary layer.

According to MA. Longo, the secondary and tertiary vortices have little impact on vortex lift and affect only slightly the position and strength of the main vortex [6]. Some Euler solutions of vortex flow at high angles of attack, use a central spatial discretisation scheme with the inclusion of special dissipative terms called "artificial viscosity" for better modelling of the creation of main vortex [6]. This is however not necessary according to K. Fuji, LB. Schiff and S. Agraval, RM. Barnett, BA. Robinson which state that it is sufficient to provide a sharp leading edge in order to obtain a realistic solution $[3,1]$. The present analysis was also conducted without the inclusion of additional dissipative terms into the solution. The flow separation at the leading edge was obtained through the modification of the airfoil involving the sharpening of the leading edge.

In the present work, the capabilities of the Euler code in the analysis of vortex flow were tested for a wing shown in figure 2 . The wing layout was close to delta; the ending had a non-zero chord due to solver constraints, which required the grid to be built of hexahedral elements (Fig 3). The grid was composed of 62 structural blocks and 1.4 million elements.

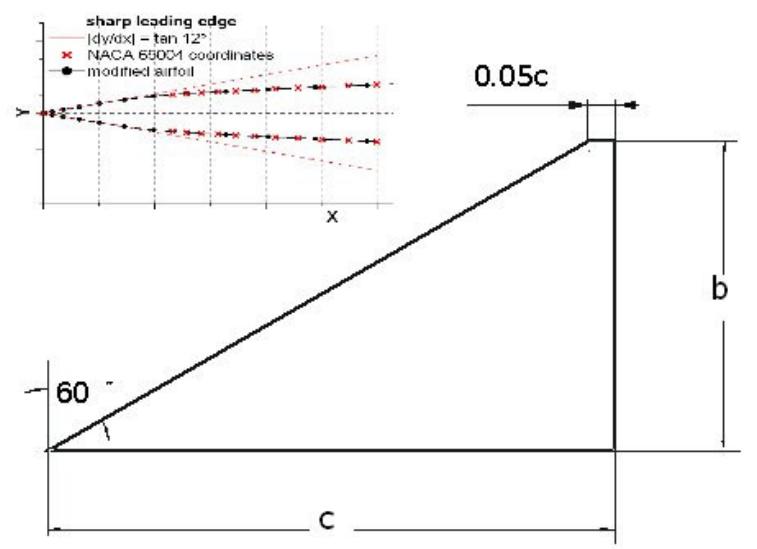

Fig 3. Geometry of the cropped-delta wing

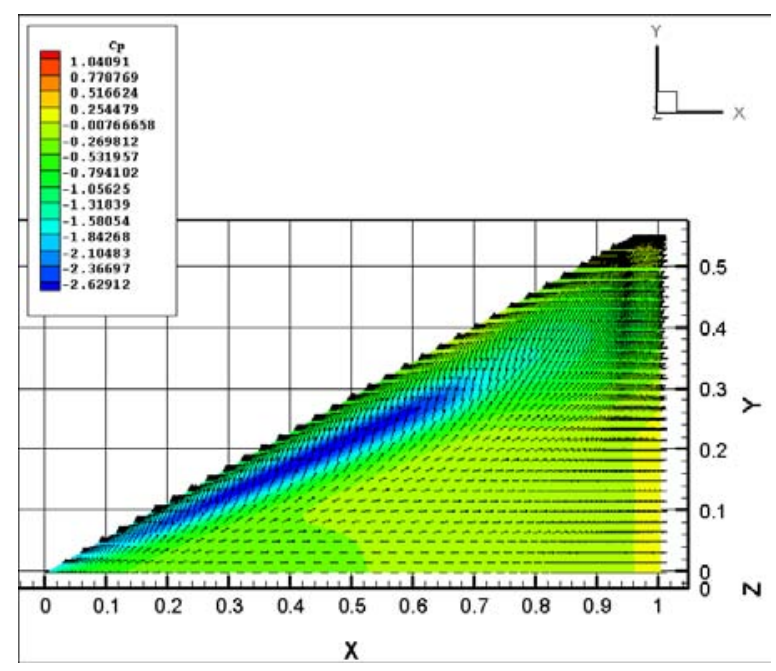

Fig 4. Pressure distribution on the upper side of the wing

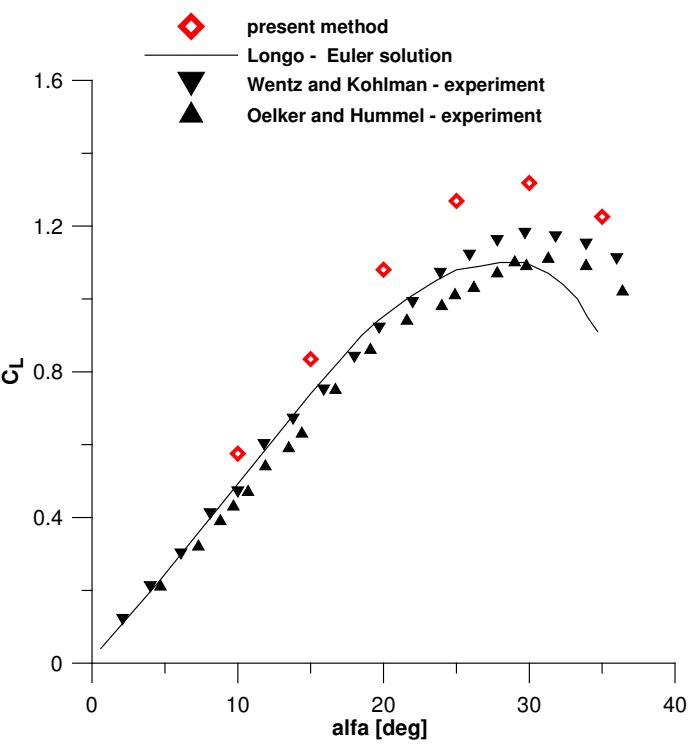

Fig 5. Comparison of $c_{\mathrm{L}}$ values computed with the present method with experimental results and another Euler solution 


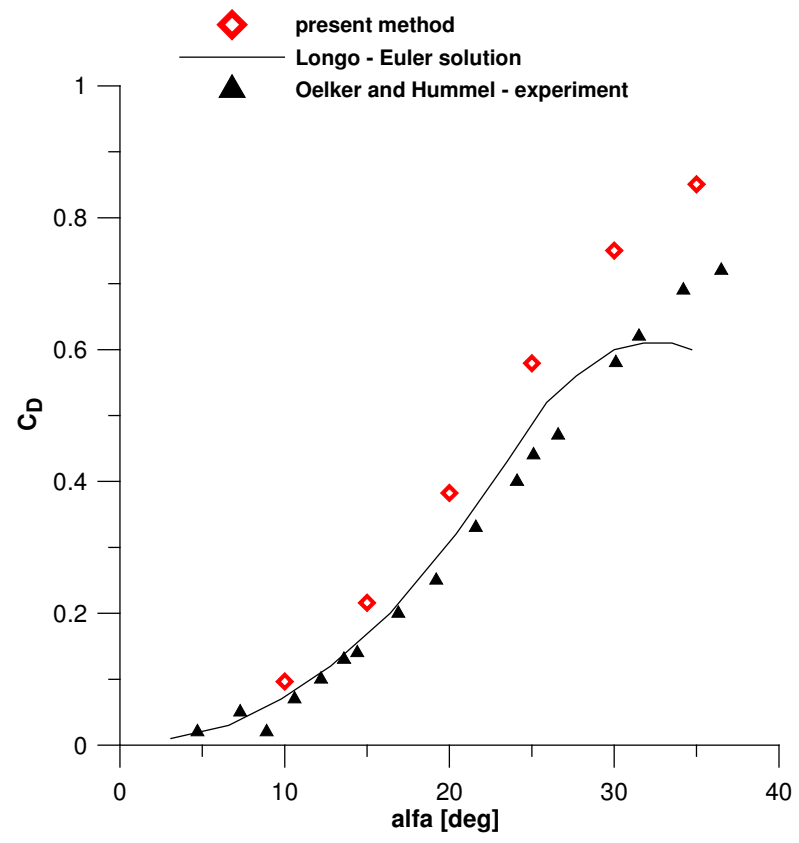

Fig 6. Comparison of $c_{D}$ values computed with the present method with experimental results and another Euler solution

The comparison of the values of the aerodynamic coefficients computed with the present method with results of experiments indicates accurate estimation of $\alpha_{C_{L \max }}$ and slight overprediction of $\frac{d c_{L}}{d \alpha}$ and $c_{L \max }$.

The $c_{D}$ values are also overpredicted, but the character of $c_{D}$ change agrees well with the experimental data. The overprediction of aerodynamic characteristics may be caused by too little distance from the wing surface to the far-field boundary surface and will be examined in future work. On the other hand, the good agreement of $\alpha_{C_{L \max }}$ with the experimental data indicates correct resolving of the position of vortex breakdown by the solver, since the loss of $c_{L}$ on this wing at high alphas is caused by the movement of the vortex breakdown point to the wing apex and loss of vortex lift. The vortex breakdown position was investigated further, with the axial velocity distribution as the criterion for vortex stability. According to MA. Longo and K. Fuji, LB. Schiff, vortex breakdown is accompanied with a rapid change in the axial vorticity distribution in a plane perpendicular to the vortex core $[6,3]$. Before the breakdown position, the distribution of the axial velocity in a plane perpendicular to the vortex axis is almost axisymmetric with maximum in the vortex centre. The vortex breakdown position is indicated by disturbances in the axi-symmetric distribution of the axial velocity, leading to the destruction of the velocity profile, such that the distribution has no longer a maximum in the vortex centre. This is illustrated in figures 7-12. Based on the abovementioned criterion, the position of vortex breakdown in this case was estimated at $\mathrm{x}=70 \%$ of the wing root chord.

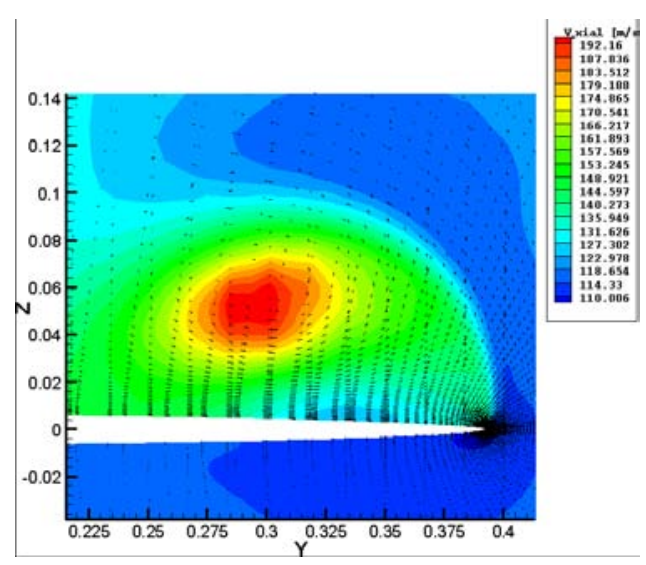

Fig 7. Distribution of axial velocity at $\mathrm{x} / \mathrm{c}=0.68$

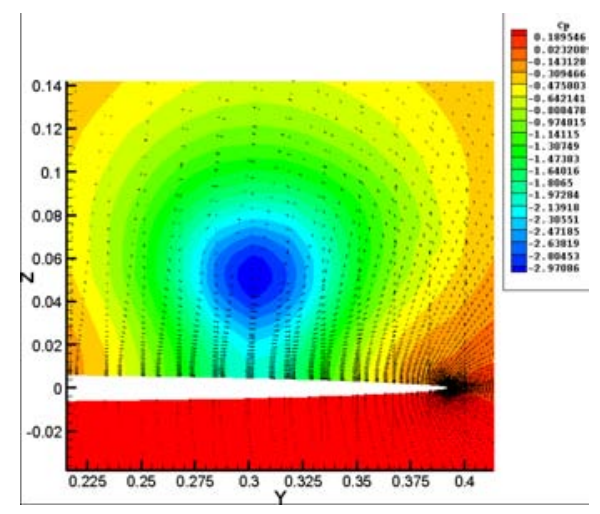

Fig 8. Distribution of pressure coefficient at $\mathrm{x} / \mathrm{c}=0.68$

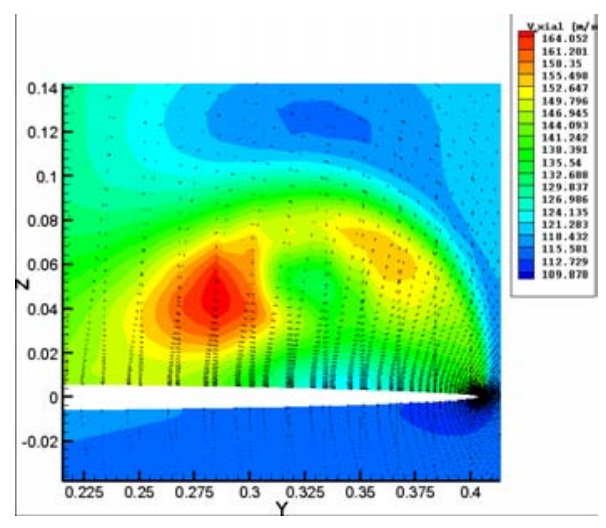

Fig 9. Distribution of axial velocity at $\mathrm{x} / \mathrm{c}=0.70$

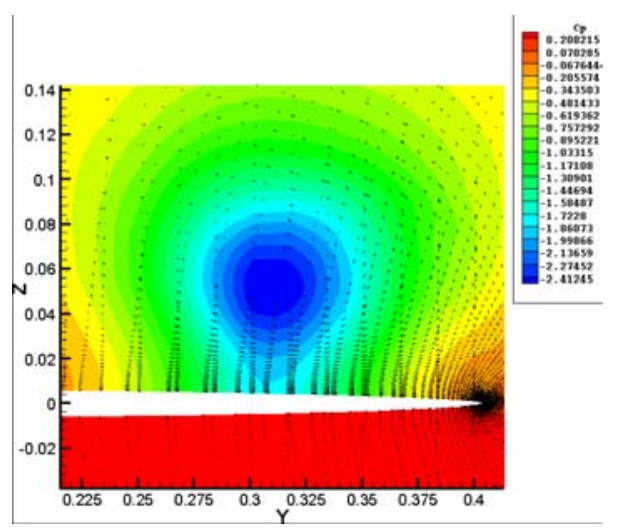

Fig 10. Distribution of pressure coefficient at $x / c=0.70$ 


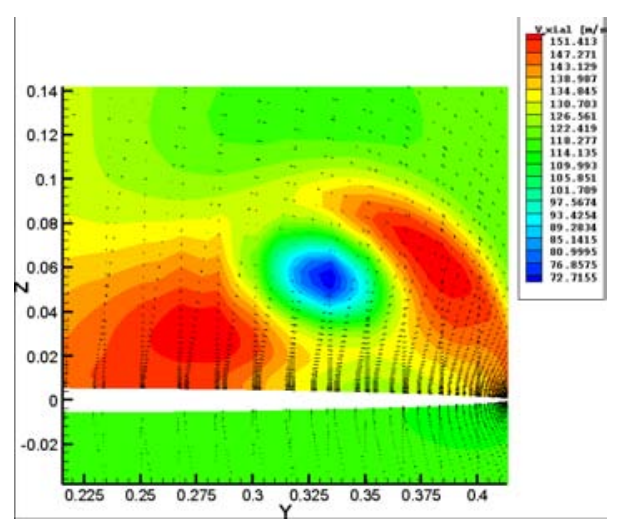

Fig 11. Distribution of axial velocity at $\mathrm{x} / \mathrm{c}=0.72$

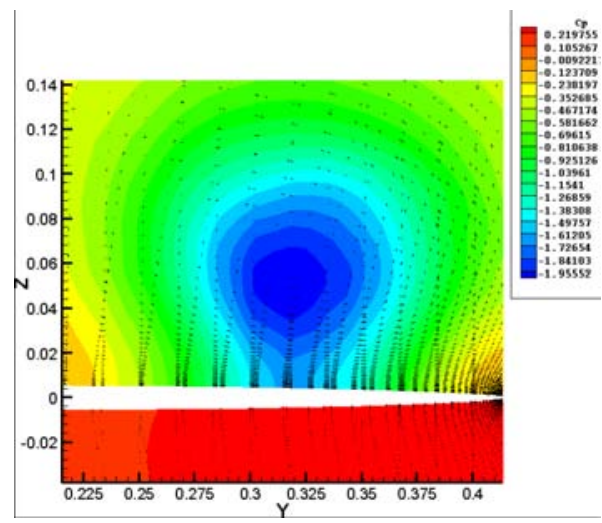

Fig 12. Distribution of pressure coefficient at $x / c=0.72$

The axial velocity and pressure coefficient distribution in two perpendicular planes containing the vortex axis was shown in figures 13 and 14 . The distributions demonstrate that vortex breakdown is a rapid process, occurring at a short distance, approximately $2 \%$ of wing root chord, and that the region of very low pressure is limited to the area upstream of the vortex breakdown.

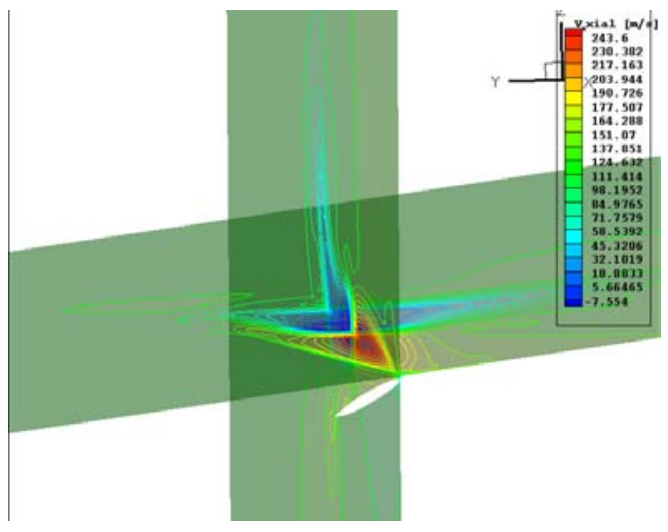

Fig 13. Distribution of axial velocity in perpendicular planes containing the vortex axis

The determination of the position of vortex breakdown is important for the aerodynamic, flight dynamics, and structural design analyses. Vortex breakdown over wings (especially asymmetric) is associated with rapid changes in many important aerodynamic derivatives-symmetric and asymmetric.
Many structural problems stemming from high unsteady aerodynamic loads near the vortex breakdown position have been reported for several aircraft, for example the F18 fighter. The big advantage of the Euler numerical solutions is that they can be used for the determination of the position of the vortex breakdown. The results of such an analysis for several values of the angle of attack are shown in figure 15 . The results obtained with the code presented were compared to experimental results and the results of another Euler solver [6]. Good agreement between the methods was obtained, except the breakdown positions near the leading and trailing edges. In the first case $\left(\alpha=30^{\circ}\right)$, the axial velocity distribution obtained at the leading edge was characteristic for a destroyed vortex core (no clear maximum). In the case for $\alpha=10^{\circ}$, the differences could be due to different shapes of the wing tip. (The reference wing was regular delta with a sharp corner while the wing investigated here had a round tip as shown in figure 3 , because of solver and mesh generator constraints).

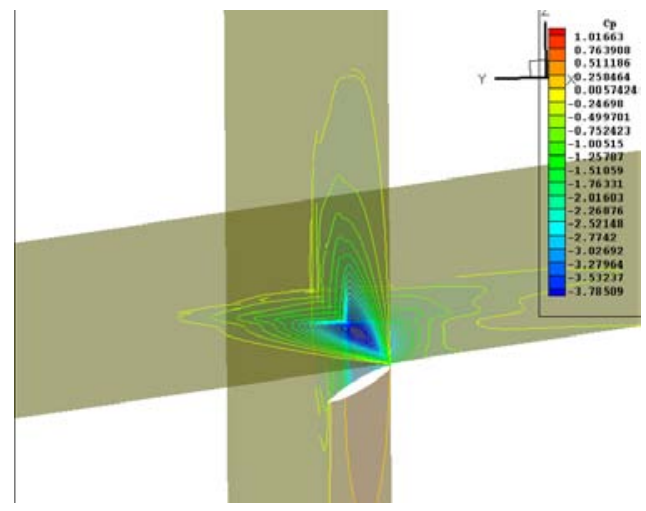

Fig 14. Distribution of pressure coefficient in perpendicular planes containing the vortex axis

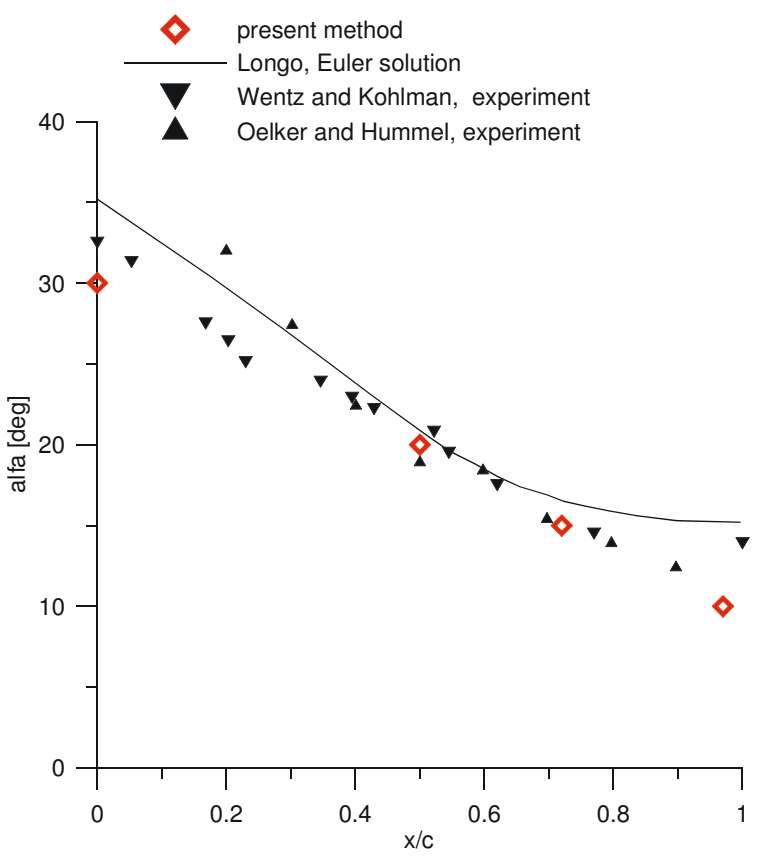

Fig 15. Comparison of vortex breakdown position computed with the present method with experimental results and another Euler solution 


\section{Unsteady flow applications-simulation of a rapid aircraft manoeuvre with the determination of aerodynamic loads using Euler equations}

The parallelization of computations provides a significant reduction in the time necessary to obtain a solution and makes it possible to conduct a coupled analysis of flight dynamics (simulation of a manoeuvre) with determination of aerodynamic loads by an Euler solver. The viscous drag forces must be introduced into the computational model from other sources, for example from engineering methods, based on wind tunnel results for similar configurations. The computations are still quite time consuming, and if run on simple machines used for engineering applications, have to be restricted to rather simple aerodynamic configurations. On the other hand, it is worthwhile to perform such an analysis in order to find the differences between the results of the coupled Flight Dynamics/Aerodynamics (FD/A) analysis and a classical Flight Dynamics model based on aerodynamic derivatives. The differences in aircraft trajectory and the peak loads acting on the airframe may provide important arguments for supplementing the classical analysis by the FD/A analysis in selected cases.

In the present case, the choice of configuration was a result of the computer equipment available for the analysis. The analysis was performed on a cluster of 42 processor PCs running the Linux operating system. Because of hardware constraints, the analysis was restricted to a symmetric configuration in symmetric flight. Additional reduction of the grid and memory size required for the analysis was accomplished through the definition of the problem as a manoeuvre of a pseudo two-dimensional configuration, consisting of a wing and horizontal tail of equal unit wing span (Fig 16). This allowed us to build a grid having only one cell across the wing span, with the symmetry boundary condition on both sides, ensuring two-dimensional character of the flow.

The geometric and mass data of the configuration is shown in the following table.

Table. The geometric and mass data of the configuration

\begin{tabular}{|r|l|}
\hline $\mathrm{c} 1=$ & $1 \mathrm{~m}$ \\
\hline $\mathrm{c} 2=$ & $0.1 \mathrm{~m}$ \\
\hline $1=$ & $3 \mathrm{~m}$ \\
\hline the tail volume parameter $=$ & 0.28 \\
\hline wing profile & NACA 0012 (both) \\
\hline mass $=$ & $200 \mathrm{~kg}$ \\
\hline $\begin{array}{r}\text { moment of inertia with } \\
\text { respect to the reference } \\
\text { point in } 0.25 \mathrm{c} 1=\end{array}$ & $112 \mathrm{~kg} \cdot \mathrm{m}^{2}$ \\
\hline
\end{tabular}

The dynamic equations of motion have the form:

$\mathrm{m}(\dot{\mathrm{U}}+\mathrm{QW})=\mathrm{X}-\mathrm{mg} \cdot \sin \Theta+\mathrm{T}_{\mathrm{X}} ;$
$\mathrm{m}(\dot{\mathrm{W}}-\mathrm{QU})=\mathrm{Z}+\mathrm{mg} \cdot \cos \Theta+\mathrm{T}_{\mathrm{Z}}$

$\mathrm{I}_{\mathrm{YC}} \dot{\mathrm{Q}}=\mathrm{M}_{\mathrm{AC}}+\mathrm{M}_{\mathrm{TC}}$

where:

$\mathrm{X}, \mathrm{Z}$, and $\mathrm{M}_{\mathrm{AC}}$ are the aerodynamic forces and moments with respect to the mass centre,

$\mathrm{T}_{\mathrm{X}}, \mathrm{T}_{\mathrm{Z}}$, and $\mathrm{M}_{\mathrm{TC}}$ are thrust force moment of thrust with respect to the mass centre,

$\mathrm{I}_{\mathrm{YC}}$ is the aircraft moment of inertia with respect to the mass centre.

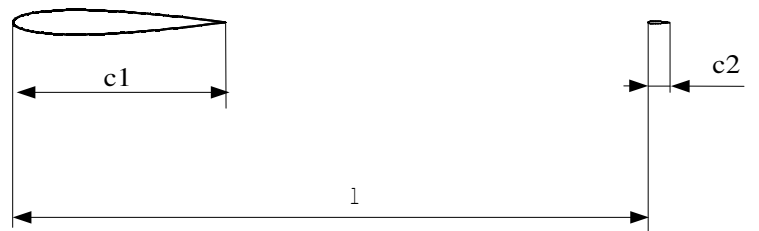

Fig 16. The two-wing configuration for the analysis of aircraft dynamics

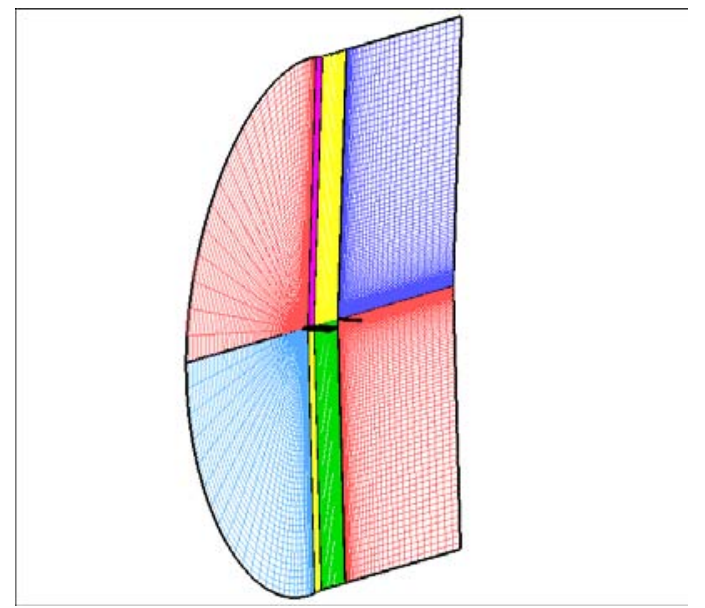

Fig 17. View of the pseudo two-dimensional grid for the configuration from Fig 16

The dynamic model of the aircraft was used with three different models of aerodynamics:

a) aerodynamic forces and moments computed with Euler equations in unsteady flow, supplemented with viscous drag forces from airfoil data,

b) aerodynamic forces and moments computed with Euler equations in steady flow, with viscous drag forces from airfoil data

c) classical aerodynamics with changes in the aerodynamic forces and moments represented through the aerodynamic derivatives:

$$
\begin{aligned}
& X=X_{0}+X_{u} \cdot u+X_{w} \cdot w+X_{q} \cdot q, \\
& Z=Z_{0}+Z_{u} \cdot u+Z_{w} \cdot w+Z_{q} \cdot q \\
& M=M_{0}+M_{u} \cdot u+M_{w} \cdot w+M_{q} \cdot q
\end{aligned}
$$

The unsteadiness of the flow was modelled by using a second order accurate-in-time discretisation scheme. In this formulation, the flow solution in each time step was 
dependent on the solutions in the two previous time steps. For each step of integrating the aircraft dynamic equations of motion in model a), there were ten steps of integrating the flow equations. The time step for the integration of the flow equations was $0.001 \mathrm{~s}$.

In model $b$ a first order accurate-in-time discretisation scheme was used, which resulted in neglecting the history of the flow. The resulting solution was obtained for a steady flow at given values of linear and angular velocity. The computational cost of variant $a$ was much higher than the cost of variant $b$; the analysis of the same manoeuvre took approximately 10 times more time than it did in variant $b$.

The aerodynamic derivatives used in the classical model of aircraft dynamics (except the X-force derivatives) were computed by solving Euler equations in the noninertial aircraft reference system using a steadyflow model. The $\mathrm{X}$ - force derivatives were computed using engineering methods to account for viscous effects.

The manoeuvre under investigation was a rapid pullout from descending flight at a $-30^{\circ}$ attitude at Mach 0.5 by the prescribed deflection of the horizontal tail (the rear airfoil). The deflection angle of the horizontal tail as a function of time is shown in figure 18.

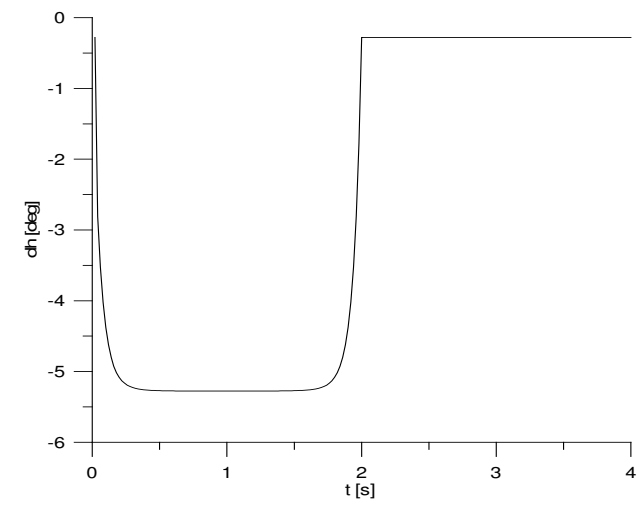

Fig 18. The prescribed deflection of horizontal tail as a function of time

The simulation of the pull-out manoeuvre was conducted for two values of stability margin. In the first case, the stability margin was equal to $20 \%$ of wing chord, and in the second case it was lowered to $5 \%$ of wing chord.

The changes in the main wing angle of attack, pitch rate, lift coefficient, and trajectory for the case with $20 \%$ stability margin is shown in figures 19-22.

The most important differences between the results of the approaches compared are smoother changes in the angle of attack and pitch rate computed with the model of unsteady flow than with the other models. The differences between the values of lift coefficient and trajectory are not significant. It can also be seen from the comparison of results for models $a$ and $b$ that accounting for the history of motion has practically no effect on the trajectory of flight. Despite lower peak values of the angle of attack and pitch rate for the model with unsteady flow, the peak values of the lift coefficient do not differ much, which suggests that disregarding the history of motion (frequent assumption in the classic approach to the analysis of aircraft dynamics) is safe for the determination of peak aerodynamic loads during the manoeuvre.

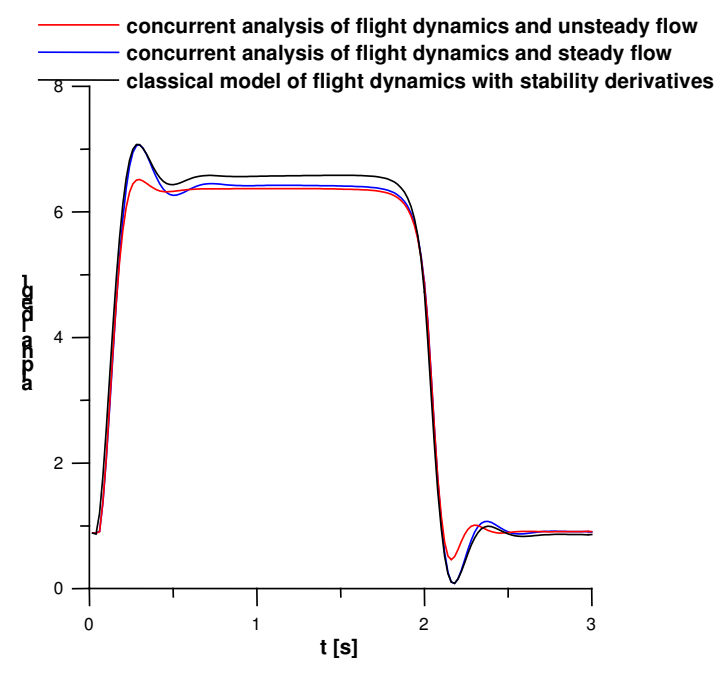

Fig 19. Change in angle of attack during the manoeuvre

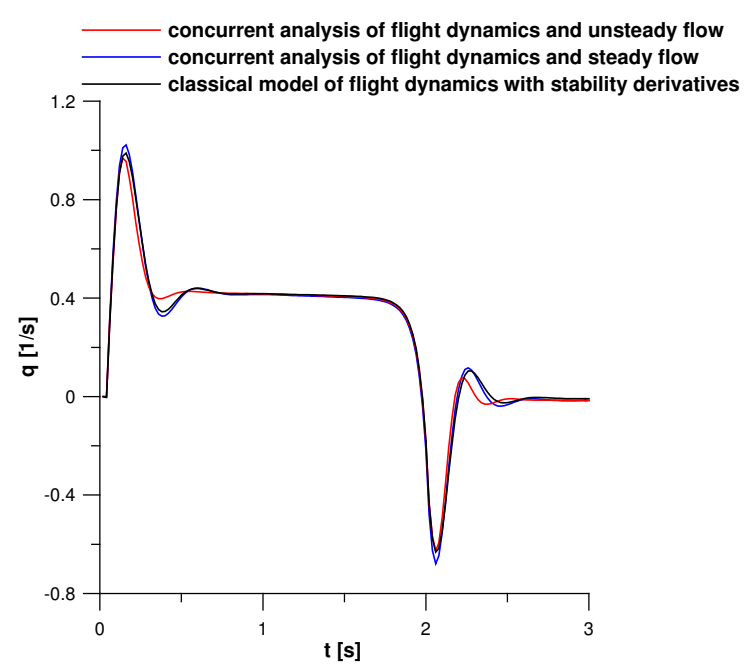

Fig 20. Change in pitch rate during the manoeuvre

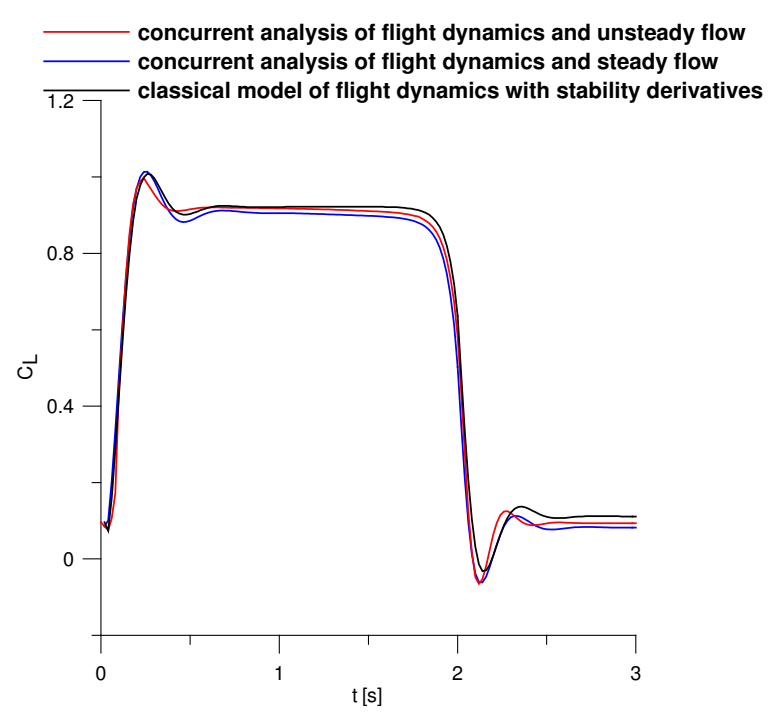

Fig 21. Change in lift coefficient during the manoeuvre 


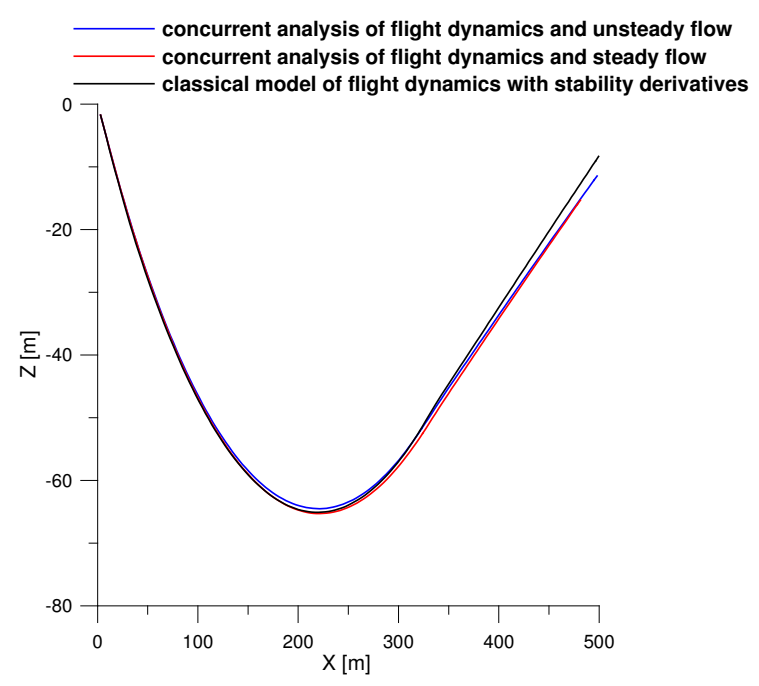

Fig 22. Flight trajectory

A similar analysis was conducted for the same manoeuvre, but with the stability margin of the aircraft model lowered to $5 \%$ of the wing chord. In addition to the three approaches to the determination of aerodynamic forces, a version of the model c) was added, for which it was assumed that the derivatives of lift and moment coefficients with respect to pitch rate (q-derivatives) and elevator effectiveness do not depend on the angle of attack. Such an assumption is sometimes applied in the simulation of aircraft manoeuvres, most often in cases when the aerodynamic derivatives are determined experimentally or by using engineering methods (ESDU Data Sheets).

The changes in the angle of attack, pitch rate, pitch angle, load factor, and the trajectory are shown in figures 23-26.

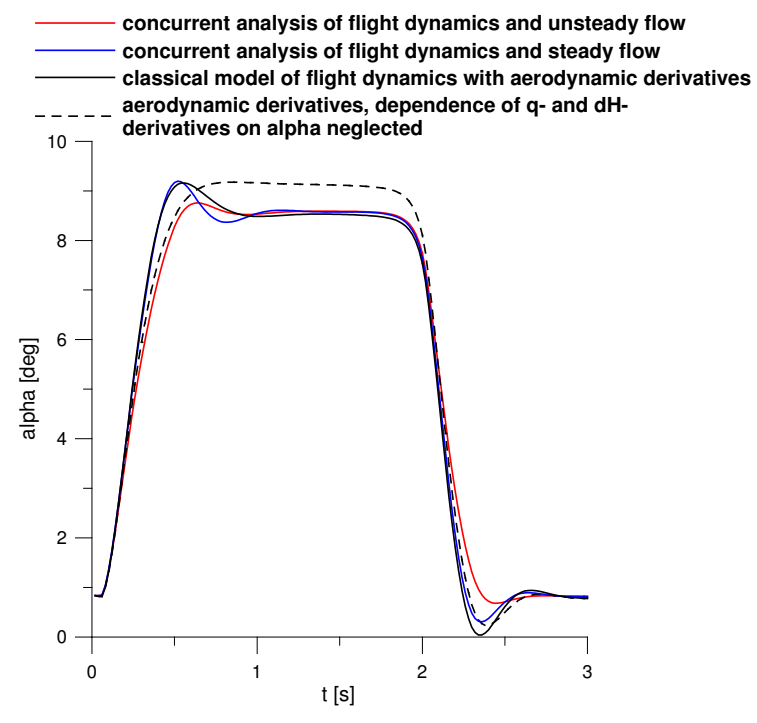

Fig 23. Change of angle of attack during the manoeuvrewith lowered stability margin

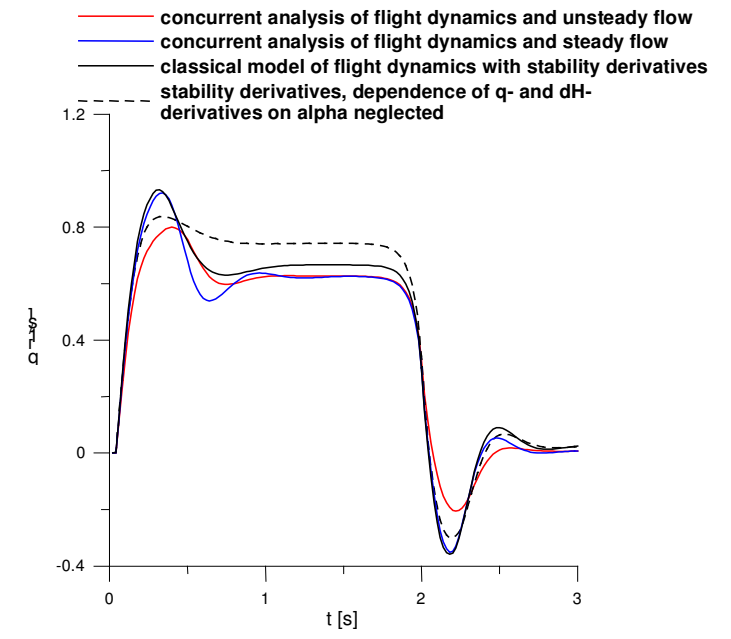

Fig 24. Change in pitch rate during the manoeuvre with lowered stability margin

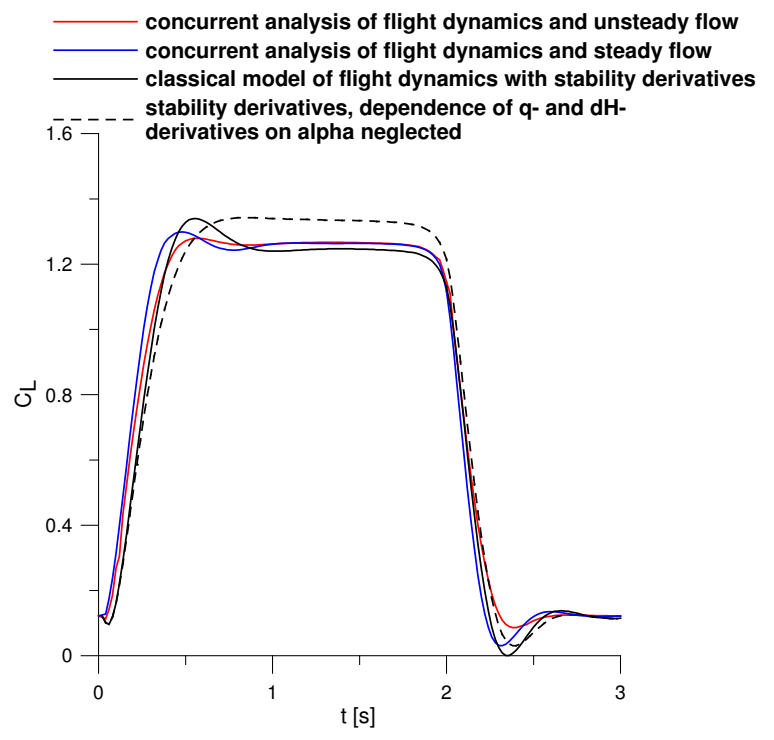

Fig 25. Change in lift coefficient during the manoeuvrewith lowered stability margin

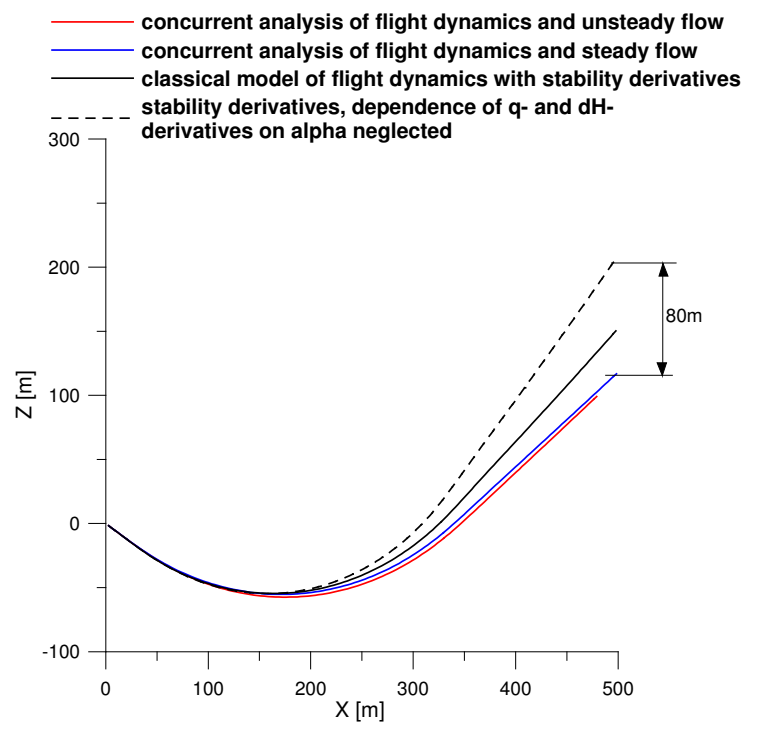

Fig 26. Flight trajectory during the manoeuvre with lowered stability margin 
The differences in the results have a character similar to the previous case. In this case, the smoothing of the changes in flight parameters for model $a$ (concurrent analysis of flight dynamics and unsteady flow) is greater than that in the case with greater stability margin. The peak values of the parameters computed with model $a$ (unsteady flow, accounting for the history of flow) are lower than those for the other models when the history of flow was disregarded. As in the previous case, accounting for the motion history had little effect on the trajectory. On the other hand, the assumption of constant values of the q-derivatives and elevator effectiveness within the range of the angles of attack considered leads to significant differences in the trajectory of the configuration. The differences between the results obtained with model $c$ (aerodynamic derivatives determined with Euler equations for steady flow) and model $b$ could be due to numerical errors in the determination of the aerodynamic derivatives and polyline approximation of the aerodynamic characteristics.

\section{Conclusions}

1. An Euler solver with the formulation of flow equations in a nonlinear reference system and the parallelization of the solution has many advantages for application in flight dynamics. It can be used to compute stability derivatives with respect to linear and angular velocities of aircraft operating in the transonic range and at high angles of attack with vortex lift. The capabilities of computer hardware available at present make it possible to conduct computations of steady characteristics and aerodynamic derivatives for complex aircraft configurations (fighters, trainers, business, etc).

2. Using a simplified aerodynamic configuration (two wings) it is possible to verify the effects of the most common simplifications applied in the simulations of aircraft manoeuvres based on quasi-steady characteristics. Neglecting the influence of the history of motion gives higher peak loads but it has little influence on flight trajectory. Disregarding the dependence of stability derivatives on the angle of attack leads to errors in the aerodynamic loads that accumulate in time and have significance, particularly on flight trajectory.

3. The differences between the results of simulation of aircraft manoeuvres (changes in the angle of attack, aerodynamic loads, and trajectory) conducted using the unsteady flow model and steady flow model grow with the decrease in the stability margin. Determination of unsteady loads during an aircraft manoeuvre may be critical for highly manoeuvrable aircraft with very low positive or with negative stability margin, particularly in cases when flight trajectory is an important result of the analysis.

4. At the present time, the analysis of aircraft dynamics with determination of unsteady aerodynamic loads by the solution of Euler equations is very costly in terms of computer resources. The analysis of the pull-out manoeuvre for the simplified aircraft configuration presented here took about 20 days of computations using four processors. Conducting such an analysis for real aircraft geometry requires at the present time massive parallelization of the solution (about 100 processors) in order to obtain the solution for a manoeuvre lasting several seconds in similar time of computations. Practical development of this method as an engineering tool still requires much progress in computational speed of the available processors.

\section{Acknowledgement}

The computations presented in this article have been supported by grant No. 5T12D03722 of the Ministry of Education and Science.

\section{References}

1. AGRAVAL, S., BARNETT, RM., ROBINSON, BA. Numerical investigation of vortex breakdown on a delta wing. AIAA Journal, vol. 30, no. 3, Mar. 1992.

2. DRIKAKIS, D., TSANGARIS, S. On the solution of the compressible Navier-Stokes equations using improved flux vector splitting methods. Applied Mathematic Modelling, vol. 17, 1993.

3. FUJI, K., SCHIFF, LB. Numerical simulations of vortical flows over a strake-delta wing. AIAA Journal, vol. 27, no. 9, Sept. 1989.

4. HITZEL, SM., Van der WEIDE, E., TREMEl, U., RIEGER, H. X31A vector high angle of attack descent Euler and Navier-Stokes simulations of unsteady manoeuvres. In Procc. of ICAS 2002 Congress.

5. LIMACHE, AC. Aerodynamic modelling using computational fluid dynamics and sensitivity equations. Doctoral dissertation. Virginia Polytechnic Institute. Blacksburg, Virginia, 2000.

6. LONGO, MA. Compressible inviscid vortex flow of a sharp edge delta wing. AIAA Journal, vol. 33, no. 4, Apr. 1995.

7. SZNAJDER, J., ŻÓŁTAK, J. Modelling of aircraft dynamics and flow during fast manouvers. In Institute of Aviation Report No. 93/BA06/P. (In Polish). 